POS PROCEEDINGS

\title{
Anisotropic Cosmic-ray Enhancement (ACRE): Case Studies of 07-Jun-2015 and 26-Aug-2018
}

\section{Agnieszka Gil*}

Siedlce University, Institute of Mathematics and Physics, Siedlce, Poland; Space Research

Center of Polish Academy of Sciences, Warsaw, Poland

E-mail: gila@uph.edu.pl

\section{Gennady A. Kovaltsov}

Ioffe Physical-Technical Institute, 194021 St. Petersburg, Russia

\section{Vladimir V. Mikhailov}

National Research Nuclear University MEPhI, Moscow, Russia

\author{
Alexandar Mishev \\ Space Climate Research Unit, University of Oulu, Finland; Sodankyla Geophysical Observatory, \\ University of Oulu, Finland
}

\section{Stepan Poluianov}

Space Climate Research Unit, University of Oulu, Finland; Sodankyla Geophysical Observatory, University of Oulu, Finland

\section{Ilya G. Usoskin}

Space Climate Research Unit, University of Oulu, Finland; Sodankyla Geophysical Observatory, University of Oulu, Finland 
We present a phenomenological study of a new type of event, named the anisotropic cosmic ray enhancement (ACRE). Such events are characterized by a small, 2-5\%, increase of the count rate of a set of polar neutron monitors (NMs) appropriately located. The first studied representative of ACRE event was observed during 12-19 UT on 07-Jun-2015. Another, smaller event took place during 3- 8 UT on 26-Aug-2018. Other possible candidates are still under consideration.

During the ACRE event of 07-Jun-2015 (Figure 1) the enhancement in cosmic-ray flux, as measured by (sub)polar NMs was very anisotropic. It was detected only by low-rigidity NMs having their asymptotic directions in the south-west quadrant in GSE coordinates. According to spaceborne measurements, there were no solar energetic particles present during that time. A neutron detector onboard the PAMELA instrument did not record any increase either, while it was located off the ACRE region. The near-Earth interplanetary conditions were quiet, with some small disturbances, causing a gradual increase in the strength of heliospheric magnetic field followed by the solar wind speed growth observed after the ACRE event. However, there was a strong solar wind shear region south-west off the Earth. We propose that the event was related to crossing of the boundary layer between two regions with different heliospheric parameters, causing a strong gradient of low-rigidity particles.

During the ACRE event on 26-Aug-2018 (Figure 2) the enhancement in cosmic-ray flux was observed even by high-rigidity, up to $8 \mathrm{GV}$ (Mexico NM), NMs, but located only in the Northern hemisphere. There was no statistically significant signal in the Southern hemisphere, even for polar NMs, implying that the event was highly anisotropic. Some disturbances in the near-Earth interplanetary conditions were recorded during this event, namely a gradual growth of heliospheric magnetic field strength, followed by the increase of solar wind speed occurring after the event.

Here we report a new type of the cosmic-ray transient event. More detailed analysis and modelling studies of the ACRE-type events are planned for forthcoming work.

36th International Cosmic Ray Conference -ICRC2019-

July 24th - August 1st, 2019

Madison, WI, U.S.A.

* Speaker. 


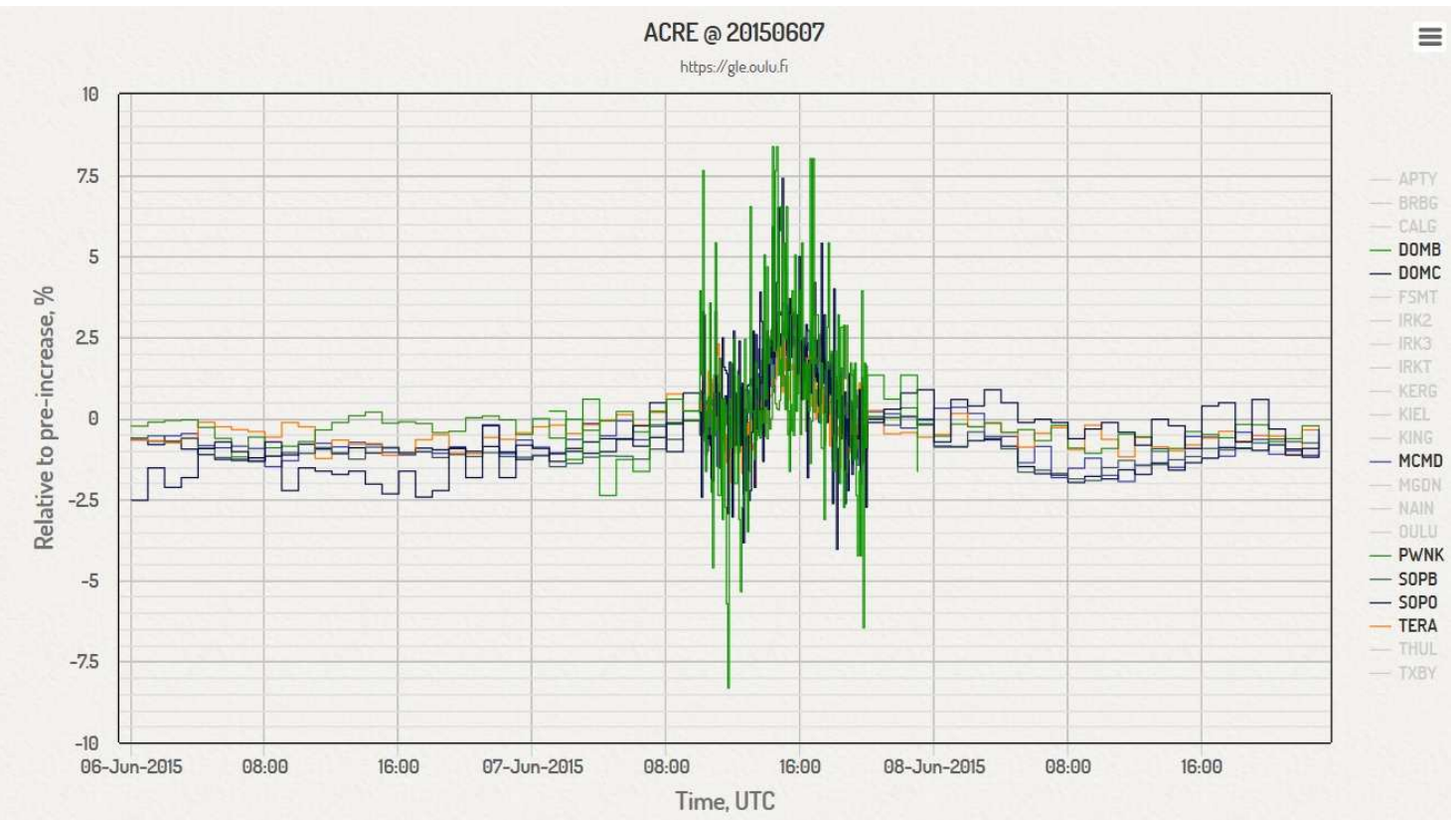

Figure 1: ACRE event from GLE data-base (https://gle.oulu.fi) on 07 June 2015 


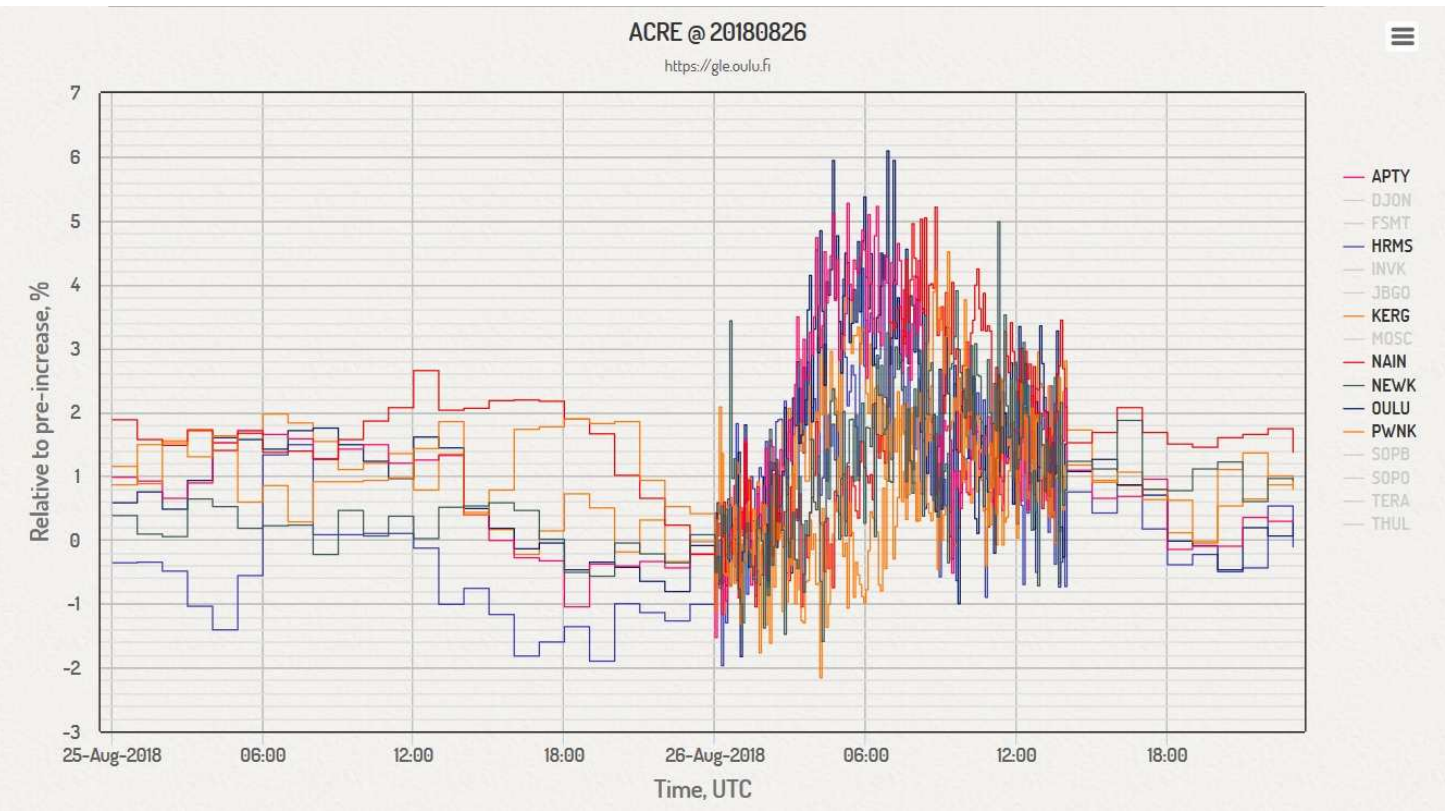

Figure 2: ACRE event from GLE data-base (https://gle.oulu.fi) on 26 August 2018 